\title{
Single-session endoscopic ultrasound-directed transgastric ERCP and percutaneous endoscopic gastrostomy (EDGE-PEG)
}

A 72-year-old man with a history of dementia, decompensated heart failure, and Roux-en-Y gastric bypass (RYGB) for obesity 30 years previously was admitted with obstructive jaundice. He also had failure to thrive, with poor oral intake and severe protein calorie malnutrition requiring enteral nutrition via a nasogastric tube.

An endoscopic ultrasound (EUS)-directed transgastric endoscopic retrograde cholangiopancreatography (ERCP) was planned for management of his obstructive jaundice, along with placement of a percutaneous endoscopic gastrostomy (PEG) for nutritional supplementation. Given the previous RYGB, placement of a
PEG tube in the gastric pouch would not have been ideal because of its anatomical location and the high risk of aspiration. Furthermore, given the bypassed small bowel, this would have precluded the optimal absorption of enteral nutrition. The EUS-guided gastro-gastrostomy created for the ERCP was fortuitous in providing access to the excluded stomach, enabling placement of the PEG in the excluded stomach.

Under EUS guidance, an endoscopic gastro-gastrostomy was performed between the gastric pouch and the excluded stomach using a $20 \times 10$-mm cautery-enhanced lumen-apposing metal stent (AXIOS; Boston Scientific, Marlborough,
Massachusetts, USA) ( Fig.1 a). The AXIOS stent forming the gastro-gastrostomy was then serially dilated from $10 \mathrm{~mm}$ to $18 \mathrm{~mm}$ using controlled radial expansion balloons (CRE; Boston Scientific) ( Fig. 1 b). The proximal flange of the stent was anchored using two endoclips (Instinct Endoscopic Clip; Cook Medical, Winston-Salem, North Carolina, USA) to prevent stent migration during the procedure ( $\vee$ Fig. $\mathbf{1} \mathbf{c}$ ).

Next, an esophagogastroduodenoscopy (EGD) was performed through the endoscopic gastro-gastrostomy and a 20-Fr pull-type PEG tube (EndoVive; Boston Scientific) was successfully placed into the excluded stomach using the stand-
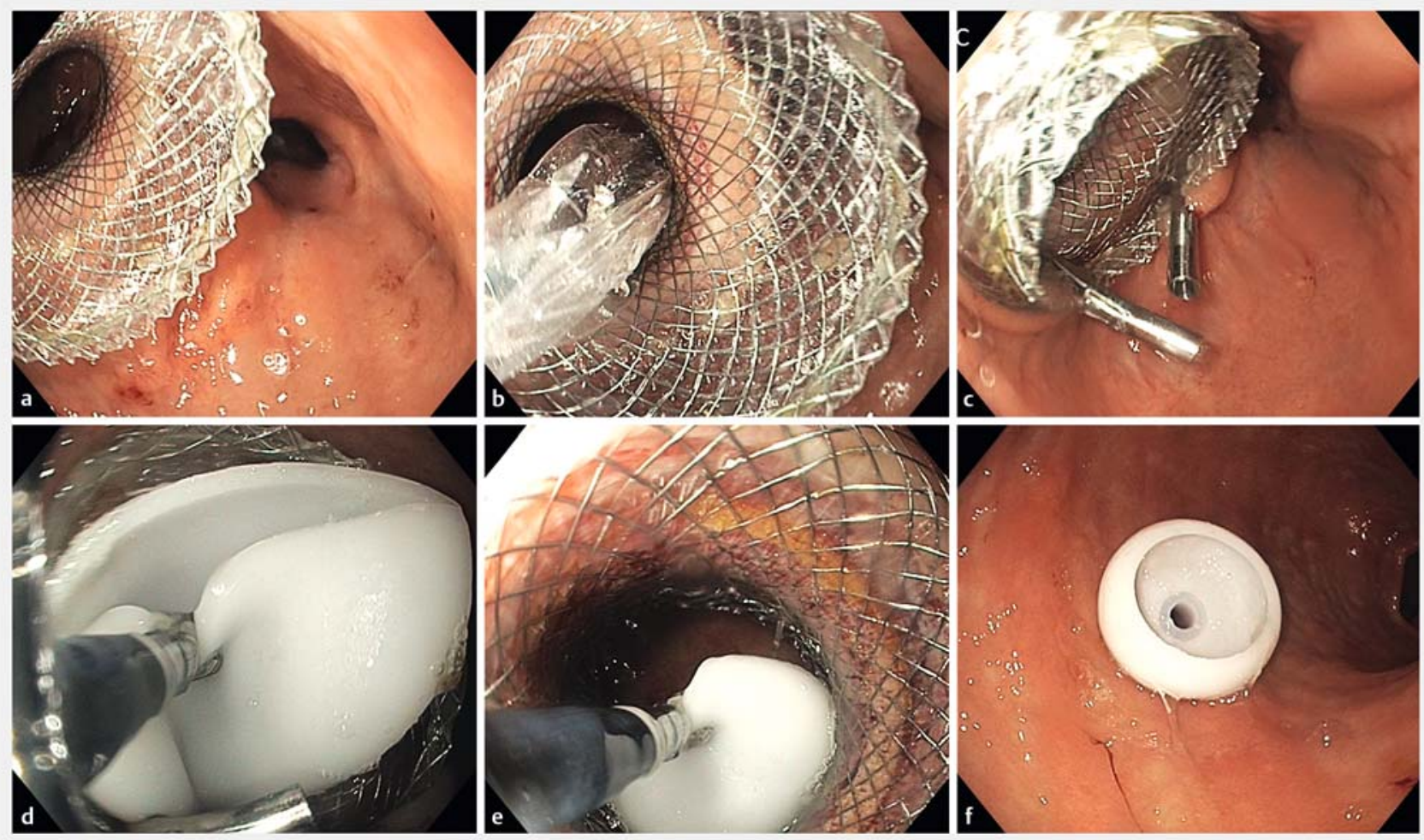

Fig. 1 Endoscopic views showing: a the gastro-gastrostomy created with a lumen-apposing metal stent (LAMS) placed between the gastric pouch and the excluded stomach; $\mathbf{b}$ balloon dilation of the gastro-gastrostomy; $\mathbf{c}$ endoclips placed to prevent stent migration during the procedure; $\mathbf{d}$, $\mathbf{e}$ the percutaneous endoscopic gastrostomy (PEG) tube bumper being guided through the LAMS using grasping forceps; $\mathbf{f}$ the successfully placed PEG tube within the excluded stomach. 


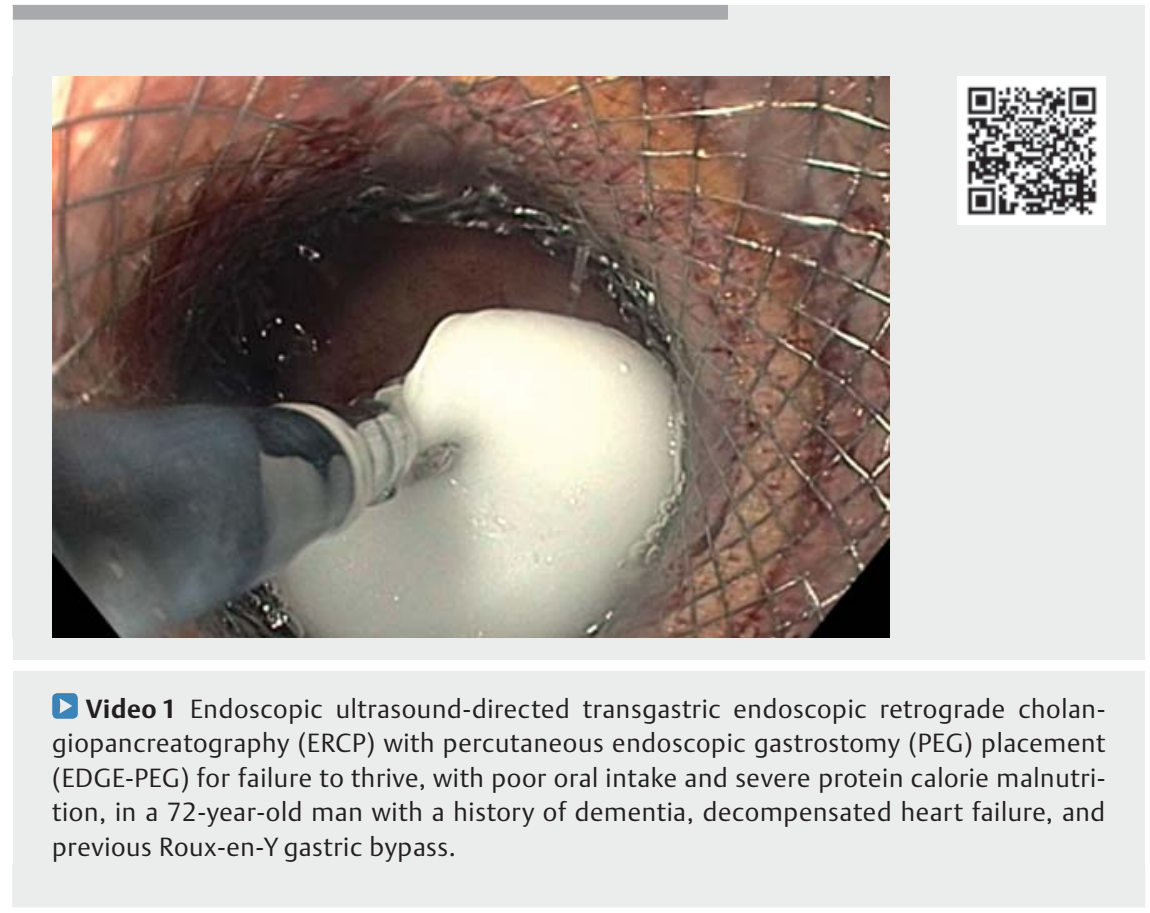

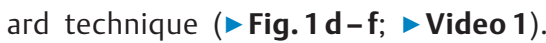
ERCP showed a distal common bile duct stricture with choledocholithiasis, which was treated with biliary sphincterotomy, stone extraction, and metal biliary stenting.

Typically, the gastro-gastrostomy is closed after completion of the planned interventions, given concerns for weight regain; however, permanent reversal may be considered in patients with progressive nutritional decline. In this case, the patient did well and was able to gain weight; however, he died from unrelated causes 3 months after the procedure.

Endoscopy_UCTN_Code_TTT_1AO_2AK

\section{Competing interests}

Tarun Rustagi is a consultant for Boston Scientific Corporation. The remaining authors declare that they have no conflict of interest.

The authors

Zain A. Sobani, Swathi Paleti, Tarun Rustagi Division of Gastroenterology and Hepatology, University of New Mexico, Albuquerque, New Mexico, USA

Corresponding author

\section{Tarun Rustagi, MD}

Division of Gastroenterology and Hepatology, University of New Mexico School of Medicine, 1 University of New Mexico, MSC10-5550, Albuquerque, NM 87131 , USA

Fax: +1-505-272-6839

tarunrustagi06@gmail.com

Bibliography

Endoscopy 2021; 53: E386-E387

DOI 10.1055/a-1308-0935

ISSN 0013-726X

published online 17.12 .2020

(c) 2020. Thieme. All rights reserved. Georg Thieme Verlag KG, Rüdigerstraße 14, 70469 Stuttgart, Germany

\section{ENDOSCOPY E-VIDEOS \\ https://eref.thieme.de/e-videos}

